\title{
Role of HER2 and Claudins in Subtypes of Urothelial Carcinoma Identified Bygata 3 and Cytokeratin5\6 Immunohistochemical Study
}

\author{
Eman A. Saad ${ }^{\text {a }}$, Kareem A. Ibrahim ${ }^{\text {b }}$ Nashwa M. Emaraa ${ }^{a}$, Amr F. El-Sebaaie ${ }^{c}$, Eman s. \\ omar $^{\text {a }}$, Samia A. youssef ${ }^{\text {a }}$
}

a Department of Pathology, Benha faculty of medicine, Benha University, Egypt. b Department of urology, Benha faculty of medicine, Benha University, Egypt. c Department of Pathology, Military Medical Academy, Cairo, Egypt

Correspondence to: Eman A. Saad, Department of pathology, Benha faculty of medicine, Benha University, Egypt.

Email:

aliasiaeman2020@yahoo.com

Received: 8 December 2020

Accepted: 11 January 2021

\begin{abstract}
:
Background: Bladder cancer is globally the ninth, most common malignancy, and the thirteenth most common cause, of cancer death, associated with high morbidity and mortality, if not treated optimally. Bladder cancers can be divided into molecular subtypes, referred to luminal and basal with distinct clinical behaviors. HER2 is one of the established therapeutic targets in many cancers.
\end{abstract} Claudins are tight junction proteins, known to modulate therapy resistance in cancer cells. Aim: This study aimed to assess Claudins and HER2 status in the context of tumor molecular subtypes, identified by GATA 3 and CK5/6 expression, that may help to select urothelial carcinoma patients, most likely to respond to immunotherapy. Materials and Methods: This retrospective study was done upon 50 cases of conventional urothelial carcinoma. GATA3, CK5/6, HER2, Claudins $1 \& 4$ and P53immunostaining were done and correlated with clinicoimmuno-pathological parameters. Results: Bladder cancers could be assigned to main intrinsic molecular subtypes, referred to luminal, basal and double negative. Basal \& double negative bladder cancers were more aggressive, when compared to luminal cancers. Positive significant statistical correlation was found between HER2, claudin1 and P53 and clinicimmuno-pathological parameters as tumor size, grade, TNM stage, LVI, tumor budding and aggressive molecular subtypes (P-value< 0.05). Negative significant statistical correlation was found between claudin 4 and fore mentioned clinico-immuno-pathological parameters (P-value< 0.05). Conclusions: The molecular subtypes of bladder cancers, HER2, 
claudin1\&4 and P53 can be used for prognostic and therapeutic stratification of bladder cancers patients, and may affect patient outcome.

Keywords: Molecular subtypes, HER2, Claudins $1 \& 4$

Abbreviations: Cytokeratin (CK) lympho-vascular invasion (LVI)

\section{Introduction}

Bladder cancer is one of common globally, increasing cancer. Worldwide, it is the 7th most common cancer in men, and the 17 th most common cancer in women, and the 9th most common in both sexes (1). Bladder cancer is the most common malignancy among Egyptian males, and previously has been attributed to Schistosoma infection, a major risk factor for squamous cell carcinoma (2).

Bladder cancers could be assigned to main intrinsic molecular subtypes, referred to luminal and basal. This may provide prognostic information, and may help to identify a subgroup of patients, with increased sensitivity to chemotherapy (3). KRT5/6 and GATA3 immunohistochemical markers, may have a great role in classifying the urothelial bladder tumors into different molecular subtypes. (4)

The HER2 is one of the epidermal growth factor receptors, which contribute to physiological mechanisms of cell proliferation, by intrinsic tyrosine-kinase activity. The overexpression of HER2 was shown in several malignancies, and it is known to affect proliferation, angiogenesis and metastasis of malignant cells. (5)

Claudins are tight junction proteins (TJs), responsible for maintaining cellular polarity, and cell-cell communication. A disruption of TJs leads to invasiveness, loss of cohesion, and lack of differentiation in cancer cells. (6)

The aim of this study, is to evaluate the IHC expression of HER2, claudins1\&4, P53 in molecular subtypes of bladder cancer, identified by GATA3 and CK5/6 expression, and correlate these results with clinicopathological data, to clarify its diagnostic, prognostic and predictive roles.

\section{Material and Methods:}

This is a retrospective study, on 50 archival formalin fixed, paraffinembedded, tissue specimens, of Egyptian patients of conventional urothelial carcinoma, collected from Benha 
Pathology Department, Faculty of Medicine, Benha University and International Medical Center, during the period from 2015 to 2019, with available demographic, and clinico-pathological data. This research plan, was approved by ethical committee, of Benha Faculty of Medicine and International Medical Center.

\section{A-Histopathological Examination:}

Hematoxylin and eosin-stained slides, on all cases, were revised by two observers simultaneously, to confirm the diagnosis. Conventional urothelial carcinoma cases, were graded, according 2016 WHO, into low grade and high grade (7). Cases were staged by, TNM staging, pT stage classified into pT0, pT1, pT2, pT3, pT4, and stage grouping into, 0, I, II, III, IV as stages0/1, were considered low stage, and stages I/III/IV were considered high stage (8).

\section{B-Immunohistochemical Procedure:}

For immunohistochemical analysis, 4micron thick sections were obtained, from formalin-fixed, paraffin-embedded tissue, blocks, on coated slides. According to manufacture instructions, antigen retrieval for Claudin1, claudin4, P53 and CK5/6 was done, by using 10
mmol/Lcitrate monohydrate buffer $(\mathrm{pH}$ 6.0), and for HER2 and GATA3, was done by using $10 \mathrm{ml}$ solution of EDTA buffer ( $\mathrm{pH}$ 9.0). The slides, were immunostained for HER2 polyclonal antibody Cat.\#A0485 (DAKO Agilent Pathology Solution), Claudin1 polyclonal antibody Cat.\#RB-9209-R7 (Thermo Fisher scientific anatomical pathology, USA), Claudin4 polyclonal antibody Cat.\#RB-9043-R7 (Thermo Fisher scientific anatomical pathology, USA), P53 monoclonal antibody Cat.\#CBL-422 (DAKO Agilent Pathology Solution), GATA3 polyclonal antibody Cat.\#YPA1589 (Chongqing Biospes Co.,Ltd ) and CK5/6 monoclonal antibody Cat.\#MA5-12429 (DAKO Agilent Pathology Solution), at a dilution of 1:100, at room temperature for 30 minutes. Immunodetection was executed, using a standard labeled streptavidin-biotin system, (Dako Cytomation, Denmark, A/S).

\section{Negative \& positive controls}

- The epidermis of apparently normal skin was used as positive control for claudin 1 \& epithelium of apparently normal colon was used as positive control for claudin4(9)

- Epithelium lining the renal collecting ducts, was used as positive 
control, for GATA3 \& apparently normal breast tissue, was used as positive control for CK5/6 (4).

- Apparently normal tonsil germinal centers B cells, was used as positive control for P53 \& HER2 positive breast cancer were used as positive control for HER2 (5)

For negative controls, omitting the primary antibody and replacing it with normal rabbit serum IgG.

\section{Immunostaining evaluation:}

Claudin1 \& Claudin4 expression, was detected as, homogeneous, brown, cytoplasmic, or membranous coloration. Immunoreactivity was assessed, based on a combined score of the extent, and intensity of staining. Scores 0-3 were assigned, according to the percentage of positive tumor cells $(0=0 \% ; 1=<25 \%$; $2=25-50 \% ; 3=>51 \%$ ), and the intensity of staining in tumo, r $(0=$ negative; $1=$ weak; $2=$ moderate; $3=$ strong). The two scores were multiplied, to give an overall score (H-score) of 0-9, of which 0 was considered negative, 1-2 was considered weak, 3-6 moderate, and 9 strong staining. Negative and weak expression was considered as low, whereas moderate and strong as high. (9)
HER2 expression was scored, using the latest ASCO/CAP guidelines published in 2016: negative $(0 / 1+)$, equivocal $(2+)$ and positive $(3+)$, with a cut-off for score $3+$, if more than $10 \%$ strongly positive, complete, membrane, staining of cells. (10)

Immunoreactivity of GATA3, was assessed, as any intensity of nuclear staining, with greater than $5 \%$ of cells, was considered positive. (11)

Immunoreactivity of CK5/6 was assessed, as a score of 0 corresponded to $0 \% ; 1$, $<1 \% ; 2,1 \%$ to $<10 \% ; 3,10 \%$ to $<33 \% ; 4$, $33 \%$ to $<66 \%$; and $5, \geqslant 66 \%$ of diffuse, homogenous, cytoplasmic, and/ or membranous staining of any intensity of tumor cells. It was determined that, scores 0,1 , and 2 indicate negativity and scores 3, 4, and 5 to indicate positivity. (12) Immunoreactivity of P53 was considered positive, if any cancer cell showed strong nuclear staining. (13)

Statistical analysis: Results were analyzed by SPSS (version 16) statistical package, for Microsoft windows. The Pearson correlation coefficient, was used for statistical analysis. $\mathrm{P}$ value $<0.05$, was considered statistically significant, and highly statistically significant when it was $<0.01$. ROC curve was also used to 
determine AUC, Sensitivity \& Specificity of all markers, as AUC $>0.7$, considered good

\section{Results}

\section{Clinico-histo-pathological results:}

There was, a significant, statistical, correlation, between pathological $\mathrm{T}$ stage, and other clinico- histo-pathological parameters, as tumor size, grade, nodal metastasis, distant metastasis, LVI, Associated CIS, Tumor budding, and focality of tumors, as P-value< $\mathbf{0 . 0 5}$.

\section{Table (1)}

\section{Immunohistochemical results:}

- Regarding molecular subtypes, the studied cases were classified according to the study of (4), as $\mathbf{5 6 \%}$ were luminal subtype ((GATA3+/CK5/6+), 32\% were basal, and $12 \%$ were double negative. Then luminal cases reclassified, regarding morphology, according to the study of (14) into, $57 \%$ of luminal cases were papillary-morphology and $43 \%$ were infiltrated non papillary morphology.

Table (2)

- There was a statistically, significant correlation, between molecular subtypes, and tumor size, tumor grade, pT stage, Stage grouping in radical cystectomy cases, lympho-vascular invasion, tumor budding, and Tumor focality (P-value< 0.05) Table (3),

- Regarding HER2 expression, there was a statistically significant correlation, between HER2 expression, and tumor size, tumor grade, pT stage, lymph node metastasis, distant metastasis in radical cystectomy, lympho-vascular invasion, tumor budding, and molecular subtypes P-valuer $\mathbf{0 . 0 5}$ Table (4), Figure (1)

- Regarding p53 expression, Positive significant statistical correlation was found between P53 and clinicoimmuno-pathological parameters, as tumor size, grade, stage, nodal metastasis, distant metastasis, LVI, Tumor budding, focality of tumors, molecular subtypes, with aggressive behavior. as P-value $<\mathbf{0 . 0 5}$. Table (4), Figure (1)

- Regarding claudins expression, Positive significant statistical correlation, was found between claudin1 and clinico-immunopathological parameters, as tumor size, grade, stage, nodal metastasis, distant metastasis, LVI, Tumor budding, focality of tumors, molecular subtypes with aggressive behavior, HER2 expression, and p53 expression, as $\mathrm{P}$ - 
value $\mathbf{0 . 0 5}$, and negative significant

clinic-immuno-pathological parameters statistical correlation was found P-value $<0.05$, Table (5), Figure (2). between claudin4, and fore mentioned

Table (1) Correlation between different clinic-pathological parameters and pathologic T stage (pT):

\begin{tabular}{|c|c|c|c|c|c|}
\hline \multirow{2}{*}{\multicolumn{2}{|c|}{ Clinico -pathological variants }} & \multirow[t]{2}{*}{ Total } & \multirow{2}{*}{\multicolumn{2}{|c|}{ pT stage }} & \multirow[t]{2}{*}{ P Value } \\
\hline & & & & & \\
\hline $\begin{array}{l}\text { Histopathological } \\
\text { variant }\end{array}$ & $\begin{array}{l}\text { Papillary } \\
\text { non papillary }\end{array}$ & $\begin{array}{l}17 \\
33\end{array}$ & $\begin{array}{c}15(88 \%) \\
3(9 \%)\end{array}$ & $\begin{array}{c}2(12 \%) \\
30(91 \%)\end{array}$ & $\begin{array}{l}\mathrm{P}=0.000 \\
\mathrm{HS}\end{array}$ \\
\hline Tumor size & $\begin{array}{l}\text { Up to } 3 \mathrm{~cm} \\
\text { More than } 3 \mathrm{~cm}\end{array}$ & $\begin{array}{l}21 \\
29\end{array}$ & $\begin{array}{c}15(71 \%) \\
3(9 \%)\end{array}$ & $\begin{array}{c}6(29 \%) \\
26(91 \%)\end{array}$ & $\begin{array}{l}\mathrm{P}=0.000 \\
\mathrm{HS}\end{array}$ \\
\hline Grade & $\begin{array}{l}\text { Low } \\
\text { High }\end{array}$ & $\begin{array}{l}18 \\
32\end{array}$ & $\begin{array}{c}15(83 \%) \\
3(9 \%)\end{array}$ & $\begin{array}{c}3(17 \%) \\
29(91 \%)\end{array}$ & $\begin{array}{l}\mathrm{P}=0.000 \\
\mathrm{HS}\end{array}$ \\
\hline $\begin{array}{l}\text { Nodal } \begin{array}{l}\text { Metastasis in } \\
\text { radical } \\
\text { cases }\end{array}\end{array}$ & $\begin{array}{l}\text { No } \\
\text { N+ }\end{array}$ & $\begin{array}{l}7 \\
19\end{array}$ & $\begin{array}{l}4(57 \%) \\
0(0 \%)\end{array}$ & $\begin{array}{c}3(43 \%) \\
19(100 \%)\end{array}$ & $\begin{array}{l}\mathrm{P}=0.01 \\
\mathrm{HS}\end{array}$ \\
\hline $\begin{array}{l}\text { Lymph } \quad \text { vascular } \\
\text { invasion }\end{array}$ & $\begin{array}{l}\text { Absent } \\
\text { Present }\end{array}$ & $\begin{array}{l}20 \\
30\end{array}$ & $\begin{array}{c}16(80 \%) \\
2(7 \%)\end{array}$ & $\begin{array}{c}4(20 \%) \\
28(93 \%)\end{array}$ & $\begin{array}{l}\mathrm{P}=0.000 \\
\mathrm{HS}\end{array}$ \\
\hline Associated CIS & $\begin{array}{l}\text { Absent } \\
\text { Present }\end{array}$ & $\begin{array}{l}28 \\
22\end{array}$ & $\begin{array}{c}17(60 \%) \\
1(5 \%)\end{array}$ & $\begin{array}{l}11(40 \%) \\
21(95 \%)\end{array}$ & $\begin{array}{l}\mathrm{P}=0.000 \\
\mathrm{HS}\end{array}$ \\
\hline Tumor focality & $\begin{array}{l}\text { Unifocal } \\
\text { Multifocal }\end{array}$ & $\begin{array}{l}32 \\
18\end{array}$ & $\begin{array}{c}18(56 \%) \\
0(0 \%)\end{array}$ & $\begin{array}{c}14(44 \%) \\
18(100 \%)\end{array}$ & $\begin{array}{l}\mathrm{P}=0.000 \\
\mathrm{HS}\end{array}$ \\
\hline Tumor Budding & $\begin{array}{l}\text { Present } \\
\text { Absent }\end{array}$ & $\begin{array}{l}25 \\
25\end{array}$ & $\begin{array}{c}17(68 \%) \\
1(4 \%)\end{array}$ & $\begin{array}{c}8(32 \%) \\
24(96 \%)\end{array}$ & $\begin{array}{l}P=0.000 \\
\text { HS }\end{array}$ \\
\hline
\end{tabular}

Table (2): Molecular subtypes of the studied cases identified by GATA3 \& CK5/6 status:

\begin{tabular}{llll}
\hline Molecular subtypes & NO. & \\
\hline Luminal (GATA3+/CK5/6+) & $28(56 \%)$ & $\begin{array}{l}\text { Luminal with papillary } \\
\text { morphology }\end{array}$ & $\begin{array}{l}\text { 16 (57\% of luminal } \\
\text { cases) }\end{array}$ \\
& & $\begin{array}{l}\text { Luminal with infiltrated non } \\
\text { papillary morphology }\end{array}$ & $\begin{array}{l}12 \text { (43\% of luminal } \\
\text { cases) }\end{array}$ \\
Basal (GATA3- /CK5/6+) & $16(32 \%)$ & \\
Double Negative (GATA3 - and CK5/6 -). & $6(12 \%)$ & \\
Total & $50(100 \%)$ & \\
\hline
\end{tabular}


Table (3) Correlation between different clinic-pathological parameters and Molecular subtypes:

\begin{tabular}{|c|c|c|c|c|c|c|c|}
\hline & & \multirow[t]{3}{*}{ Total } & \multicolumn{4}{|c|}{ Molecular subtypes } & \multirow[t]{3}{*}{ P Value } \\
\hline & & & $\begin{array}{c}\text { Luminal - } \\
\text { papillary } \\
\text { morphology }\end{array}$ & $\begin{array}{c}\text { Luminal - } \\
\text { infiltrated non } \\
\text { papillary } \\
\text { morphology }\end{array}$ & Basal & $\begin{array}{c}\text { Double } \\
\text { Negative }\end{array}$ & \\
\hline & & & NO & NO & NO & No & \\
\hline \multirow{2}{*}{$\begin{array}{l}\text { Histopathological } \\
\text { variant }\end{array}$} & Papillary & 17 & $16(94 \%)$ & $0(0 \%)$ & $1(6 \%)$ & $0(0 \%)$ & 0.001 \\
\hline & $\begin{array}{l}\text { non } \\
\text { papillary }\end{array}$ & 33 & $0(0 \%)$ & $12(36 \%)$ & $15(45 \%)$ & $6(18 \%)$ & HS \\
\hline \multirow[t]{2}{*}{ Grade } & Low grade & 18 & $15(83 \%)$ & $3(17 \%)$ & $0(0 \%)$ & $0(0 \%)$ & 0.001 \\
\hline & High grade & 32 & $1(3 \%)$ & $9(28 \%)$ & $16(50 \%)$ & $6\left(19 \% \_\right)$ & HS \\
\hline \multirow[t]{5}{*}{ pT stage } & $\mathbf{N} \quad \mathbf{p T a}$ & 8 & $8(100 \%)$ & $0(0 \%)$ & $0(0 \%)$ & $0(0 \%)$ & 0.000 \\
\hline & MI pT1 & 11 & $7(64 \%)$ & $3(27 \%)$ & $1(9 \%)$ & $0(0 \%)$ & HS \\
\hline & MI pT2 & 21 & $1(5 \%)$ & $6(29 \%)$ & $10(48 \%)$ & $4(19 \%)$ & \\
\hline & pT3 & 9 & $0(0 \%)$ & $3(33 \%)$ & $4(44 \%)$ & $2(22 \%)$ & \\
\hline & pT4 & 1 & $0(0 \%)$ & $0(0 \%)$ & $1(100 \%)$ & $0(0 \%)$ & \\
\hline \multirow{2}{*}{$\begin{array}{l}\text { Stage grouping in } \\
\text { radical } \\
\text { cystectomy cases }\end{array}$} & $\begin{array}{l}\text { Low stage } \\
(0 \& I)\end{array}$ & 7 & $3(43 \%)$ & $3(43 \%)$ & $1(14 \%)$ & $0(0 \%)$ & $\begin{array}{l}0.004 \\
\text { HS }\end{array}$ \\
\hline & $\begin{array}{l}\text { High stage } \\
\text { (II,III\&IV) }\end{array}$ & 19 & $1(5 \%)$ & $5(26 \%)$ & $8(42 \%)$ & $5(26 \%)$ & \\
\hline \multirow[t]{2}{*}{ Associated CIS } & Present & 22 & $0(0 \%)$ & $2(9 \%)$ & $14(64 \%)$ & $6(27 \%)$ & 0.002 \\
\hline & Absent & 28 & $16(57 \%)$ & $10(36 \%)$ & $2(7 \%)$ & $0(0 \%)$ & HS \\
\hline \multirow[t]{2}{*}{ Tumor budding } & Present & 25 & $1(4 \%)$ & $4(16 \%)$ & $14(56 \%)$ & $6(24 \%)$ & 0.000 \\
\hline & Absent & 25 & $15(60 \%)$ & $8(32 \%)$ & $2(8 \%)$ & $0(0 \%)$ & HS \\
\hline \multirow[t]{2}{*}{ Tumor focality } & Unifocal & 32 & $16(50 \%)$ & $10(31 \%)$ & $6(19 \%)$ & $0(0 \%)$ & 0.000 \\
\hline & Multifocal & 18 & $0(0 \%)$ & $2(11 \%)$ & $10(56 \%)$ & $6(33 \%)$ & HS \\
\hline
\end{tabular}


Table (4) Correlation between clinic-immuno-pathological Variants and HER2 and P53 Expression:

\begin{tabular}{|c|c|c|c|c|c|c|c|c|c|}
\hline \multirow{2}{*}{\multicolumn{2}{|c|}{$\begin{array}{l}\text { Clinicoimmunopathological } \\
\text { variants }\end{array}$}} & \multirow{2}{*}{\multicolumn{2}{|c|}{ Total }} & \multicolumn{2}{|c|}{ HER2 expression } & \multirow{2}{*}{$\begin{array}{l}\mathbf{P} \\
\text { Value }\end{array}$} & \multicolumn{2}{|c|}{ P53 expression } & \multirow{2}{*}{$\begin{array}{l}\mathbf{P} \\
\text { Value }\end{array}$} \\
\hline & & & & Negative & Positive & & Negative & Positive & \\
\hline \multirow{2}{*}{$\begin{array}{l}\text { Histopatho } \\
\text { logical } \\
\text { variant }\end{array}$} & Papillary & \multicolumn{2}{|l|}{17} & $15(88 \%)$ & $2(12 \%)$ & \multirow{2}{*}{$\begin{array}{l}\mathrm{P}= \\
0.108\end{array}$} & $13(76 \%)$ & $4(24 \%)$ & \multirow{2}{*}{$\begin{array}{l}P=0.00 \\
\text { 0 } \\
\text { HS }\end{array}$} \\
\hline & non papillary & 33 & & $16(48 \%)$ & $17(52 \%)$ & & $8(24 \%)$ & $25(76 \%)$ & \\
\hline \multirow[t]{2}{*}{ Grade } & Low & 18 & & $\begin{array}{l}18 \\
(100 \%)\end{array}$ & $0(0 \%)$ & $\begin{array}{l}\mathrm{P}= \\
0.001\end{array}$ & $14(78 \%)$ & $4(22 \%)$ & \multirow{2}{*}{$\begin{array}{l}P=0.00 \\
5 \\
\text { HS }\end{array}$} \\
\hline & High & 32 & & $13(41 \%)$ & $19(59 \%)$ & HS & $7(22 \%)$ & $25(78 \%)$ & \\
\hline \multirow[t]{7}{*}{ pT stage } & NMI & pTa & 8 & $8(100 \%)$ & $0(0 \%)$ & $\mathrm{P}=$ & $7(88 \%)$ & $1(12 \%)$ & \multirow{7}{*}{$\begin{array}{l}P=0.00 \\
1 \\
\text { HS }\end{array}$} \\
\hline & & pT & 1 & 11 & $0(0 \%)$ & 0.01 & $6(55 \%)$ & $5(45 \%)$ & \\
\hline & & 1 & 1 & $(100 \%)$ & & $\mathrm{S}$ & & & \\
\hline & MI & $\mathrm{pT}$ & 2 & $9(42 \%)$ & $12(58 \%)$ & & $6(29 \%)$ & $15(71 \%)$ & \\
\hline & & 2 & 1 & & & & & & \\
\hline & & $\begin{array}{l}\mathrm{pT} \\
3\end{array}$ & 9 & $3(33 \%)$ & $6(67 \%)$ & & $2(22 \%)$ & $7(78 \%)$ & \\
\hline & & & 1 & $0(0 \%)$ & $1(100 \%)$ & & $0(0 \%)$ & $1(100 \%)$ & \\
\hline \multirow{4}{*}{$\begin{array}{l}\text { Staging } \\
\text { group in } \\
\text { radical } \\
\text { cystectomy } \\
\text { cases } \\
\text { Associated } \\
\text { CIS }\end{array}$} & $\begin{array}{l}\text { Low- } \\
\text { stage }(0 / I)\end{array}$ & 7 & & $7(100 \%)$ & $0(0 \%)$ & $\begin{array}{l}\mathrm{P}=0.00 \\
6\end{array}$ & $5(71 \%)$ & $2(29 \%)$ & $\begin{array}{l}P=0.00 \\
6\end{array}$ \\
\hline & $\begin{array}{l}\text { High } \\
\text { stage(II/III/IV } \\
\text { ) }\end{array}$ & 19 & & $5(26 \%)$ & $\begin{array}{l}14 \\
(74 \%)\end{array}$ & HS & $8(42 \%)$ & $11(58 \%)$ & HS \\
\hline & Absent & 28 & & $18(65 \%)$ & $10(35 \%)$ & $\mathrm{P}=$ & $19(68 \%)$ & $9(32 \%)$ & \multirow{2}{*}{$\begin{array}{l}P=0.00 \\
\text { O } \\
\text { HS }\end{array}$} \\
\hline & Present & 22 & & $13(59 \%)$ & $9(41 \%)$ & 0.371 & $2(9 \%)$ & $20(91 \%)$ & \\
\hline \multirow[t]{2}{*}{$\begin{array}{l}\text { Tumor } \\
\text { budding }\end{array}$} & Absent & 25 & & $24(96 \%)$ & $1(4 \%)$ & $\begin{array}{l}\mathrm{P}= \\
0.01\end{array}$ & $18(72 \%)$ & $7(28 \%)$ & \multirow{2}{*}{$\begin{array}{l}P=0.00 \\
1 \\
\text { HS }\end{array}$} \\
\hline & Present & 25 & & $7(28 \%)$ & $18(72 \%)$ & $\mathrm{S}$ & $3(12 \%)$ & $22(88 \%)$ & \\
\hline Tumor & Unifocal & 32 & & $22(70 \%)$ & $10(30 \%)$ & $\mathrm{P}=$ & $19(59 \%)$ & $13(41 \%)$ & $P=0.00$ \\
\hline focality & Multifocal & 18 & & $9(50 \%)$ & $9(50 \%)$ & 0.09 & $2(11 \%)$ & $16(86 \%)$ & $\begin{array}{l}1 \\
\text { HS }\end{array}$ \\
\hline \multirow[t]{4}{*}{$\begin{array}{l}\text { molecular } \\
\text { subtypes }\end{array}$} & $\begin{array}{l}\text { Luminal } \\
\text { papillary }\end{array}$ & 16 & & $15(94 \%)$ & $1(6 \%)$ & $\begin{array}{l}\mathrm{P}= \\
0.022\end{array}$ & $12(75 \%)$ & $4(25 \%)$ & $\begin{array}{l}P=0.00 \\
0\end{array}$ \\
\hline & $\begin{array}{l}\text { Luminal- } \\
\text { infiltrated }\end{array}$ & 12 & & $1(8 \%)$ & $11(92 \%)$ & $\mathrm{S}$ & $6(50 \%)$ & $6(50 \%)$ & \multirow[t]{3}{*}{ HS } \\
\hline & Basal & 16 & & $5(94 \%)$ & $1(6 \%)$ & & $3(13 \%)$ & $13(87 \%)$ & \\
\hline & $\begin{array}{l}\text { Double- } \\
\text { Negative }\end{array}$ & 6 & & $0(0 \%)$ & $6(100 \%)$ & & $0(0 \%)$ & $6(100 \%)$ & \\
\hline
\end{tabular}




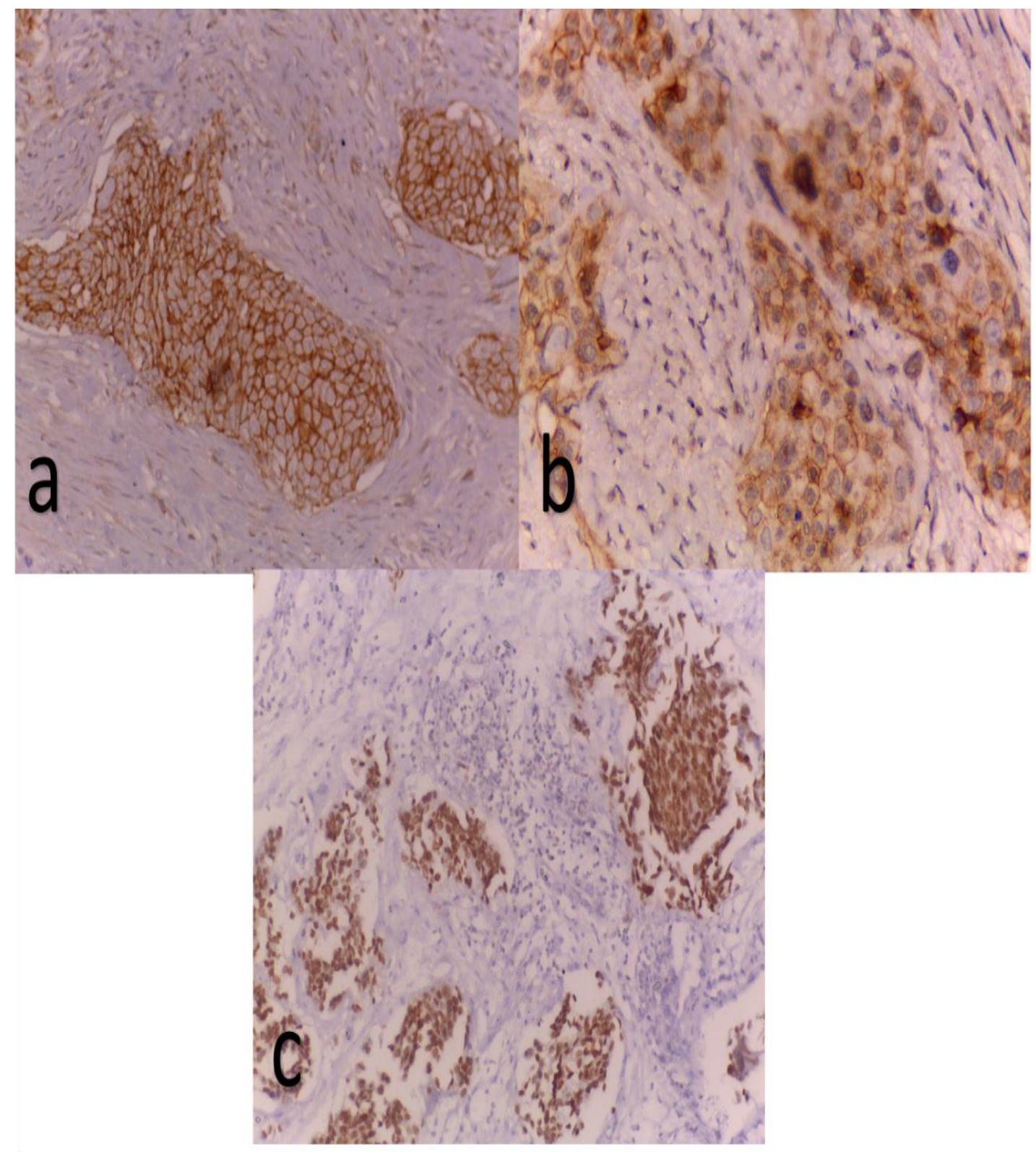

Figure (1): a) high grade invasive non papillary urothelial carcinoma showing strong complete membrane staining forHER2 in more than 10\% of tumor cells score 3(ABCX200). b) high grade invasive non papillary urothelial carcinoma showing incomplete membrane staining for HER2 expression in more than $10 \%$ of tumor cells score 2(ABCX400). c) high grade invasive urothelial carcinoma with evident LVI showing strong positive nuclear P53 staining in tumor cells and tumor emboli (ABCX200) 
Table (5) Correlation between clinic-immuno-pathological Variants and Claudin1\&claudin4 expression:

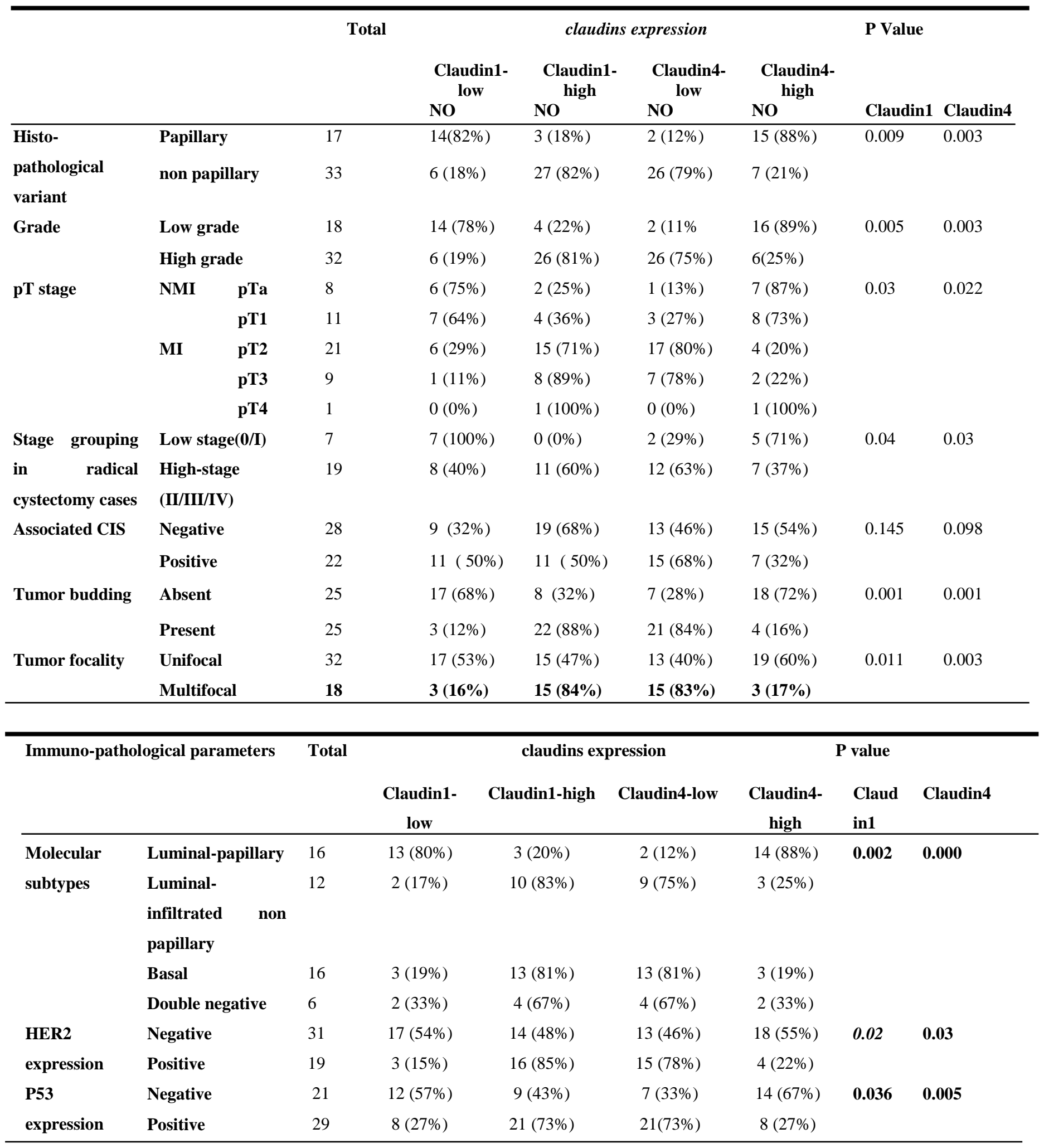




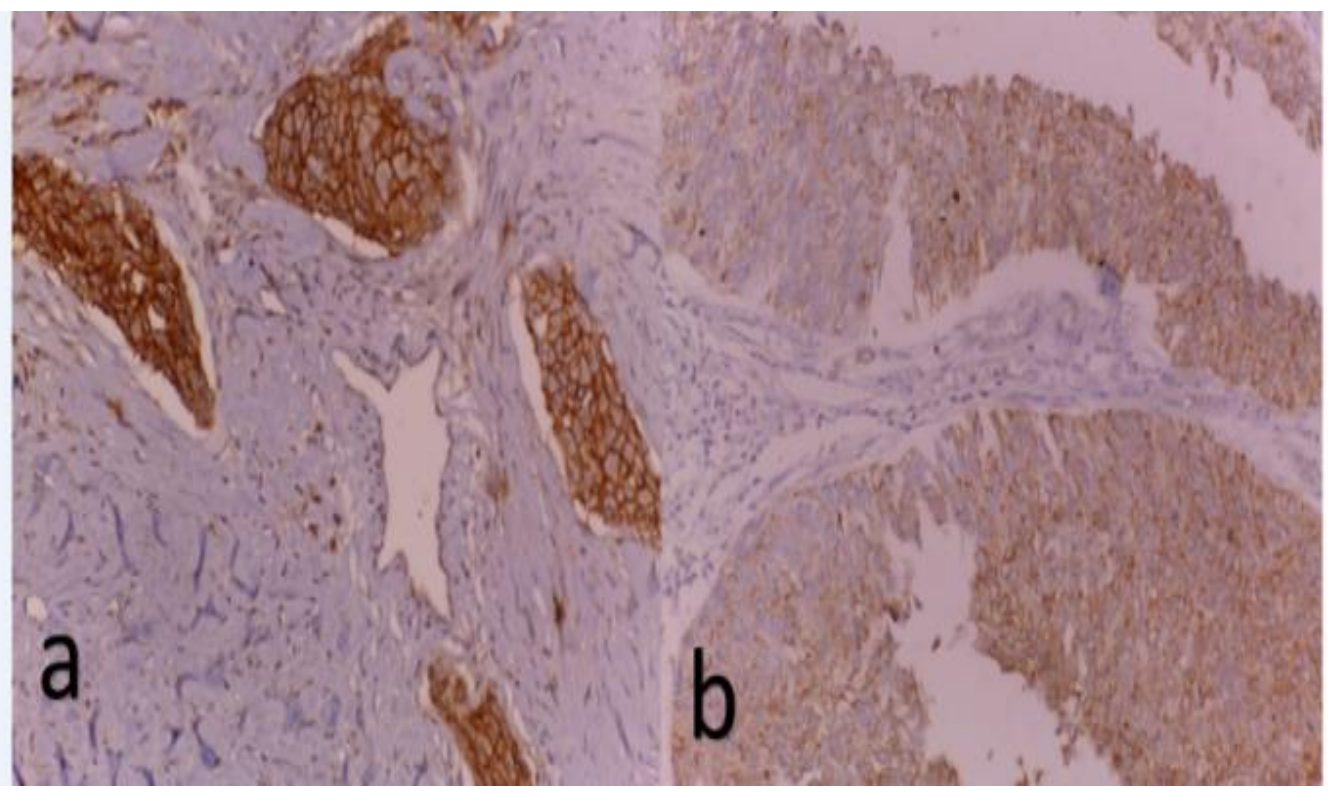

Figure (2): a) High grade, urothelial carcinoma, showing strong membranous expression in more than $50 \%$ of tumor cells, claudin1 expression, in tumor cells (claudin1 high, score9), (ABCX200). b) low grade, papillary urothelial carcinoma, showing moderate membranous, \&cytoplasmic claudin 4 , expression in more than $25 \%$ of tumor cells, score 4(ABCX200).

Table (6) Statistical determination of sensitivity and specificity of HER2, Claudin1, Claudin4 and P53S biomarkers results and Molecular subtypes by using ROC curve analysis:

\begin{tabular}{llccc}
\hline Molecular type & Marker & AUC & Sensitivity (\%) & Specificity (\%) \\
\hline Luminal - papillary & HER2 & 0.296 & 6.2 & 53 \\
& Claudin1 & 0.197 & 18.8 & 20.6 \\
& Claudin4 & 0.820 & 90.91 & 67.44 \\
Luminal -infiltrated non & P53 & 0.257 & 25.0 & 26.5 \\
papillary morphology & HER2 & 0.770 & 81.82 & 67.44 \\
& Claudin1 & 0.645 & 73.7 & 57.1 \\
Basal & Claudin4 & 0.375 & 25.0 & 50.0 \\
& P53 & 0.447 & 50.0 & 39.5 \\
& HER2 & 0.297 & 6.2 & 52.9 \\
Double negative & Claudin1 & 0.656 & 81.2 & 50.0 \\
& Claudin4 & 0.314 & 18.8 & 43.1 \\
& P53 & 0.717 & 85.7 & 63.64 \\
& HER2 & 0.875 & 95.6 & 70.9 \\
\end{tabular}

AUC for claudin 4 in luminal papillary subtype is0.820. AUC for HER2 in luminal non-papillary subtype is 0.770 . AUC for p53 in Basal subtype is 0.717 . AUC for HER2 \&p53 in double negative subtype are $0.875 \& 0.738$ respectively. 

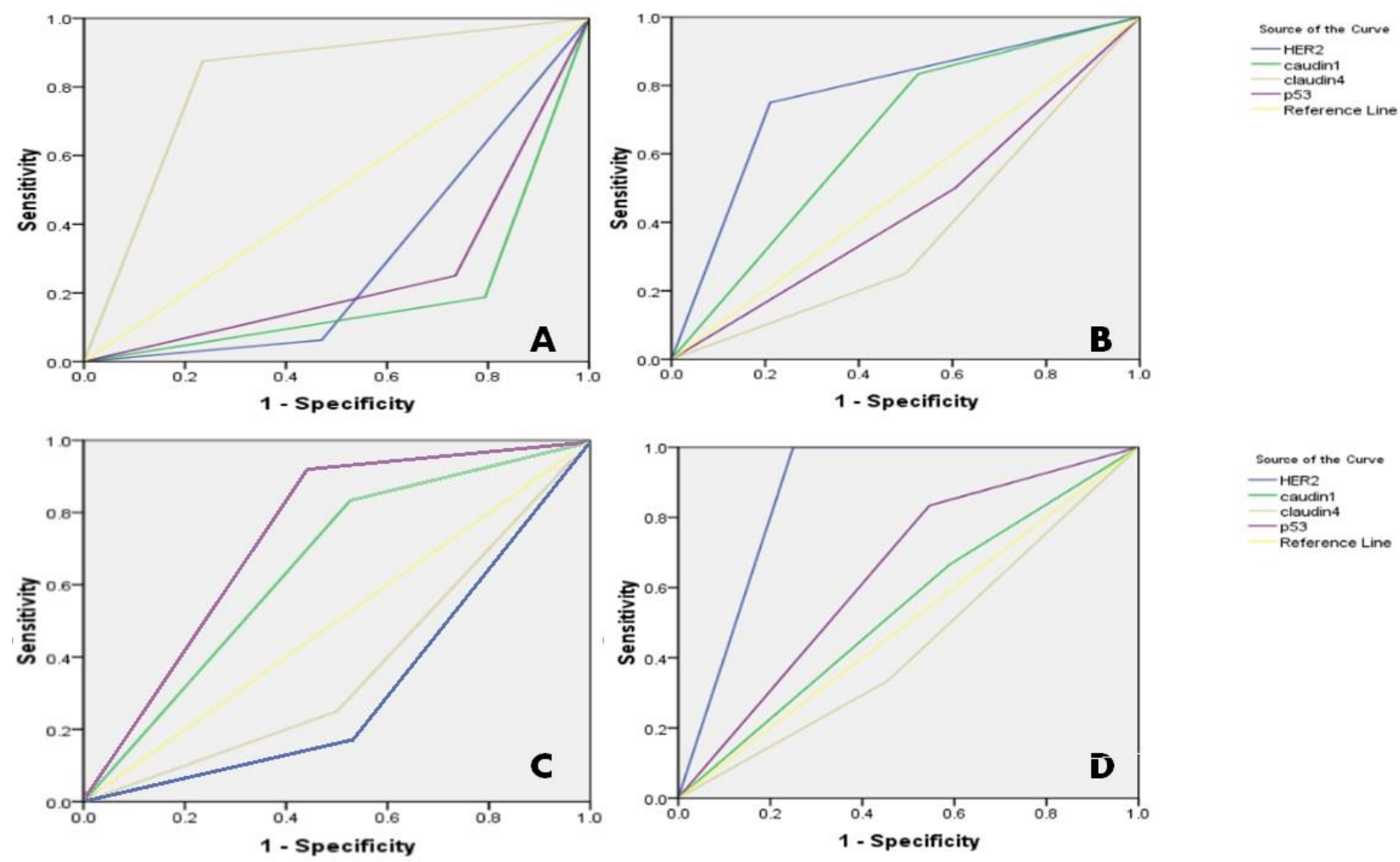

Figure (3): Diagnostic performance, using ROC curve analysis for, A) luminal papillary subtype B) luminal infiltrated subtype, C) Basal subtype, D) Double negative subtype

\section{Discussion}

Bladder cancer, is one of common increasing cancer worldwide. In Egypt, it is the third, most common cancer, in both sexes (15).

This retrospective study, was carried on 50 cases, of conventional transitional cell carcinoma (TCC) of urinary bladder. The mean age of the studied cases, was 61.34 years. This agrees with the study of (16), the male to female ratio of the studied cases, was 3.5:1. This agrees with the study of (17).

Conventional TCC cases, in this study were $34 \%$ papillary transitional cell carcinoma, and $66 \%$ infiltrating-non papillary transitional cell carcinoma, this in line with previous study (18). In disagreement with previous study (19), who had majority of patients with superficial tumors. According to 2016 
WHO grading system, the studied cases, were (36\%) low grade and (64\%) high grade. This finding is in agreement, with previous study on bladder urothelial cancer (7), which found that, (32\%) of the cases were low grade, and (68\%) were high grade. While previous study (20), found, (57\%) of the cases, were low grade, and $(43 \%)$ were high grade.

Regarding muscle invasion, (38\%) of the cases were non-muscle invasive and (62\%)were muscle invasive, this is in agreement with previous study (8), which stated that, most of urinary bladder TCC cases, present in an advanced stage. While, previous study (19), showed that, majority of cases were non muscle invasive. The differences in results of the present study, and other studies regarding grade and muscle invasion, may be contributed to differences in patient awareness, and late diagnosis in Egyptian patients. (21)

On basis of IHC analysis, using GATA3 and CK5/6 markers, the studied cases were classified into, three molecular subtypes, $56 \%$ were Luminal, $32 \%$ were basal subtype and $12 \%$ were Double Negative subtype. This distribution of molecular subtypes, is nearly similar to the study of (22). Then luminal cases reclassified, regarding morphology, according to the study of (14) into, 57\% were luminal papillary and $43 \%$ were luminal infiltrated morphology. There was a statistically, significant, correlation, between molecular subtypes, and tumor grade, pT stage, TNM stage in radical cystectomy cases, LVI, associated CIS, tumor budding, and tumor focality as (Pvalues 0.05) revealing that, Luminalpapillary subtype was associated with the best prognosis, while Basal and Double Negative subtypes had a more aggressive behavior, with a tendency to early invasion and metastases. These results were in line with previous study (4) which showed that the molecular subtypes were significantly associated with tumor grade \&stage and luminal subtype was associated with more favorable outcome when compared to basal subtype.

Immuno-histochemical evaluation of HER 2 expression, revealed a statistically significant correlation between HER2 expression, and tumor size, tumor grade, pT stage, TNM stage in radical cystectomy cases, LVI, tumor budding, and molecular subtypes as (P-valuer 0.05). These results were in parallel with study of (23), (24), (25). The poor prognostic impact of Her2/neu in the studied cases, may be contributed to the role of HER2, as one of receptor tyrosine 
kinase proto-oncogenes, that enhances cancer cell proliferation, motility, invasion and metastasis (26). However, (27) on his study on gastric carcinoma, found that, HER2 expression was not related to gastric cancer patient prognosis. This differences may be contributed to different tissue on which this study done.

This study also revealed a statistically significant correlation, between HER2 expression and molecular subtypes, (P value $=0.022)$ showing that, tumors with a luminal molecular subtype, had a significantly higher rate of Her2 alterations, than those of the basal subtype This result was in line with previous study (10), which suggested that, HER2 activity was also associated with subtype status.

The p53 gene, is the most frequently altered gene, in human cancers, and P53 mutations have been associated with genomic instability, and hence progressive development, of further mutations. (28).

Immuno-histochemical evaluation of P53 expression, revealed a significant statistical correlation between P53 and clinico-immuno-pathological parameters, as tumor size, grade, stage, LVI, Tumor budding, focality of tumors, and molecular subtypes with aggressive behavior as Pvalue $\mathbf{0 . 0 5}$. This result was in parallel with study of (29), (30), (13). However, previous study (31) suggested that p53 expression was not significantly associated with the stage or grade of bladder cancer.

This study also revealed, a statistically, significant, correlation, between p53 expression, and molecular subtypes ( $\mathrm{P}$ value $=\mathbf{0 . 0 0 0})$, as all cases with Double Negative molecular subtypes, showed positive nuclear expression of p53. This was in line with previous studies (4) and (32). That study revealed, there was no a significant, statistical, correlation between p53 expression, and HER2 expression, $(\mathrm{P}$ value $=0.08)$.

Immuno-histochemical evaluation of Claudin1\&4 expression, revealed that a positive significant statistical correlation was found, between claudin 1 and clinicoimmuno-pathological parameters as tumor size, grade, stage, LVI, Tumor budding, focality of tumors, as $\mathrm{p}$ value $<\mathbf{0 . 0 5}$, and a negative significant statistical correlation was found, between claudin 4 and fore mentioned clinic-immuno-pathological parameters, as P-value< 0.05. These previous results were in line with the study of (9). These results could be explained by, the role of Claudin 1 in directly promoting epithelial mesenchymal transition (EMT), through its interaction 
with, defined EMT-related transcription factors, and signaling pathways. while Claudin-4 expression increased the barrier function of tight junctions, and inhibited the migration, and invasion of cancer cells (33)

In contrast with previous studies (34), (35)

and (36) which reported that, increased expression of Claudin-4, together with claudin3 and KI67, was correlated to advanced stage and poor prognosis, and loss of Claudin1 expression, together with claudin7, was associated with high grade, and stage tumors. This different results may have contributed to, claudins action was influenced and modulated by interaction with other claudins members as claudins $3 \& 7$ and other proliferating genes as KI67.

This study also revealed a statistically significant correlation between claudins (1\&4) expression and molecular subtypes $(\mathrm{P}$ value $=0.002 \& 0.000$ for claudin 1 and claudin4 respectively) as $81 \%$ of cases with basal molecular subtypes, showed high expression of claudin1, and low expression of claudin 4 , and $67 \%$ of double negative cases, showed high expression of claudin1, and low expression of claudin4. These results were in line with, previous studies (37), and (4), which stated that, major subset of basal-like, and doublenegative tumors, showed downregulation of claudin target genes (claudins 3, 4, and 7). And in line with the study of (38), on ductal breast carcinoma, who demonstrated that majority of luminal subtype showed low claudin-1 expression, and triple negative cases, showed high claudin 1 expression.

This study also revealed a statistically significant correlation between claudins (1\&4) expression and HER2 expression (P value $=0.02 \& 0.037$ for claudin 1 and claudin4 respectively). This results were in line with study done by (39) on breast carcinoma, who demonstrated that, absence HER2 expression, was associated with low expression claudin4. And in line with previous study (38), that demonstrated that, claudin-1 overexpression, was associated with HER2 enriched, breast carcinoma. This study also revealed, a statistically, significant, correlation, between p53 expression, and claudins (1\&4), as $\boldsymbol{P}$ value $=0.036 \& 0.005$ for claudin 1 and claudin 4 respectively. This result was in line with previous report (40) which stated that expression of p53 and claudin1 was significantly increased in SCC of vulva. 
Regarding, results of ROC analysis, Claudin4 may be considered, better biomarker, in prediction of Luminal papillary subtype, HER2 may be considered, better biomarker, in prediction of Luminal infiltrated subtype, p53 may be considered, better biomarker in prediction of basal subtype. HER2, P53 and, claudin1 may be considered, better biomarkers in prediction of double negative subtype.

\section{Conclusion}

Molecular subtypes of bladder cancers, and expressions of HER2, claudin $1 \& 4$ and P53 can be used for, prognostic, and therapeutic purpose of $\mathrm{BC}$ patients, that may affect patient outcome.

\section{References}

1-Moch H, Humphrey PA, Ulbright TM. Who classification of tumours of the urinaryt system and male genital organs. In Who classification of tumours of the urinaryt system and male genital organs 2016 (pp. 356-356).

2-Mokhtar N, Salama A, Badawy O, Khorshed E, Mohamed G, Ibrahim M. Cancer Pathology Registry A 12-year Registry 2000-2011. National Cancer Institute. 2016;13:192-208.

3-Weyerer V, Schneckenpointner R, Filbeck T, Burger M, Hofstaedter F, Wild PJ, etal. Immunohistochemical and molecular characterizations in urothelial carcinoma of bladder in patients less than 45 years. Journal of Cancer. 2017;8(3):323.
4-Dadhania V, Zhang M, Zhang L, Bondaruk J, Majewski T, Siefker-Radtke A, etal. Metaanalysis of the luminal and basal subtypes of bladder cancer and the identification of signature immunohistochemical markers for clinical use. EBioMedicine. 2016 Oct 1;12:10517.

5-Sarode VR, Xiang QD, Christie A, Collins R, Rao R, Leitch AM, etal. Evaluation of HER2/neu status by immunohistochemistry using computer-based image analysis and correlation with gene amplification by fluorescence in situ hybridization assay: A 10year experience and impact of test standardization on concordance rate. Archives of Pathology and Laboratory Medicine. 2015 Jul;139(7):922-8.

6-Zhao X, Zou Y, Gu Q, Zhao G, Gray H, Pfeffer LM, etal. Lentiviral vector mediated claudin1 silencing inhibits epithelial to mesenchymal transition in breast cancer cells. Viruses. 2015 Jun;7(6):2965-79.

7-Moch H, Humphrey PA, Ulbright TM. Who classification of tumours of the urinaryt system and male genital organs. InWho classification of tumours of the urinaryt system and male genital organs 2016 Aug 14(6): 356-356.

8-Amin MB, Greene FL, Edge SB, Compton CC, Gershenwald JE, Brookland RK,etal . The eighth edition AJCC cancer staging manual: continuing to build a bridge from a populationbased to a more "personalized" approach to cancer staging. CA: a cancer journal for clinicians. 2017 Mar;67(2):93-9.

9-Kokenek-Unal TD, Coban I, Oguz-Erdogan AS, Seneldir H, Gurcay N, Alper M. Differential Expression of Claudin-1, Claudin-3, and 
Claudin-4 in Bladder Lesions. Journal of Cancer and Tumor International. 2015 Aug 14:117-27.

10-Kiss B, Wyatt AW, Douglas J, Skuginna V, Mo F, Anderson S, etal. Her2 alterations in muscleinvasive bladder cancer: Patient selection beyond protein expression for targeted therapy. Scientific reports. 2017 Feb 16;7(1):1-0.

11-Inoue S, Mizushima T, Fujita K, Meliti A, Ide H, Yamaguchi S, Fushimi H, Netto GJ, Nonomura N, Miyamoto H. GATA3 immunohistochemistry in urothelial carcinoma of the upper urinary tract as a urothelial marker and a prognosticator. Human Pathology. 2017 Jun 1;64:83-90.

12-Maeda I, Tajima S, Kanemaki Y, Tsugawa K, Takagi M. Use of immunohistochemical analysis of CK5/6, CK14, and CK34betaE12 in the differential diagnosis of solid papillary carcinoma in situ from intraductal papilloma with usual ductal hyperplasia of the breast. SAGE Open $\quad$ Medicine. 2018 Nov;6:2050312118811542.

13-Stec R, Cierniak S, Lubas A, Brzóskowska U, Syryło T, Zieliński H, etal. Intensity of Nuclear Staining for Ki-67, p53 and Survivin as a New Prognostic Factor in Non-muscle Invasive Bladder Cancer. Pathology \& Oncology Research. 2020 Apr;26(2):1211-9.

14-Kamat AM, Matulay JT. Advances in risk stratification of bladder cancer to guide personalized medicine. F1000Research. 2018 Jan 1;7:1137.

15-Saginala K, Barsouk A, Aluru JS, Rawla P, Padala SA, Barsouk A. Epidemiology of Bladder Cancer. Medical Sciences. 2020 Mar;8(1):15.

16-Gunlusoy B, Ceylan Y, Degirmenci T, Aydogdu O, Bozkurt IH, Yonguc T, etal. The potential effect of age on the natural behavior of bladder cancer: Does urothelial cell carcinoma progress differently in various age groups?. The Kaohsiung journal of medical sciences. 2016 May 1;32(5):261-6.

17-Rambau PF, Chalya PL, Jackson K. Schistosomiasis and urinary bladder cancer in North Western Tanzania: a retrospective review of 185 patients. Infectious agents and cancer. 2013 Dec;8(1):1-6.

18-Babjuk M, Böhle A, Burger M, Capoun O, Cohen D, Compérat EM, etal. EAU guidelines on non-muscle-invasive urothelial carcinoma of the bladder: update 2016. European urology. 2017 Mar 1;71(3):447-61.

19-Cumberbatch MG, Jubber I, Black PC, Esperto F, Figueroa JD, Kamat AM, etal. Epidemiology of bladder cancer: a systematic review and contemporary update of risk factors in 2018. European urology. 2018 Dec 1;74(6):784-95.

20- Barua SK, Das N, Baruah SJ, Rajeev TP, Bagchi PK, Sarma D, etal. Correlation of epidermal growth factor receptor score with prognostic variables in transitional cell carcinoma of urinary bladder among northeastern Indian population. International Surgery Journal. 2018 Aug 25;5(9):3083-8.

21-El-Bolkainy MN, Nouh MA, Gouda I, ElBolkainy TN, Badawy OM. Pathology of cancer, Vol. I, 2013.

22-Blinova E, Enikeev D, Roshchin D, Samyshina E, Deryabina O, Tertychnyy A, etal. RelapseFree Survival and PD-L1 Expression in First High-and Low-Grade Relapsed Luminal, Basal and Double-Negative P53-Mutant NonMuscular Invasive Bladder Cancer Depending on Previous Chemo-and Immunotherapy. Cancers. 2020 May;12(5):1316. 
23-Hammam O, Nour HH, Mosaad M, Akl M, Khalil H, Al Ganzory H, etal. The clinical significance of HER2 protein amplification/expression in urinary bladder lesion. Arab journal of urology. 2015 Jun 1;13(2):146-52.

24-Zhao J, Xu W, Zhang Z, Song R, Zeng S, Sun $\mathrm{Y}$, etal. Prognostic role of HER2 expression in bladder cancer: a systematic review and metaanalysis. International urology and nephrology. 2015 Jan 1;47(1):87-94.

25-Nedjadi T, Al-Maghrabi J, Assidi M, Dallol A, Al-Kattabi H, Chaudhary A, etal. Prognostic value of HER2 status in bladder transitional cell carcinoma revealed by both IHC and BDISH techniques. BMC cancer. 2016 Dec 1;16(1):653.

26-Charfi S, Mnif H, Ellouze S, Mhiri MN, Boudawara-Sellami T. Immunohistochemical expression of HER2 in urothelial bladder carcinoma and its correlation with p53 and p63 expression. Journal of Microscopy and Ultrastructure. 2013 Jun 1;1(1-2):17-21.

27-Grabsch H, Sivakumar S, Gray S, Gabbert HE, Müller W. HER2 expression in gastric cancer: rare, heterogeneous and of no prognostic valueconclusions from 924 cases of two independent series. Analytical Cellular Pathology. 2010 Jan 1;32(1-2):57-65.

28-Wang CC, Tsai YC, Jeng YM. Biological significance of GATA3, cytokeratin 20, cytokeratin 5/6 and p53 expression in muscleinvasive bladder cancer. PloS one. 2019 Aug 30;14(8):e0221785.

29-Mitra AP. Molecular substratification of bladder cancer: moving towards individualized patient management. Therapeutic advances in urology. 2016 Jun;8(3):215-33.
30-Al Chalabi R, Salih SM, Saad S, Jawad H. Expression of p53 and p21 in bladder carcinoma of Iraqi patients. Journal of Biological ResearchBollettino della Società Italiana di Biologia Sperimentale. 2019 Jun 24;92(1).

31-Bodoor K, Al-Ghabkari A, Matalka I, Haddad Y, Alkhateeb A, Jaradat S, etal. Assessment of p53 mutations, expression and prognosis in bladder cancer patients from Jordan: Identification of novel deletion mutations in the DNA-binding domain. Meta Gene. 2017 Jun $1 ; 12: 33-42$.

32-Lee E, Collazo-Lorduy A, Castillo-Martin M, Gong Y, Wang L, Oh WK, Galsky MD, Cordon-Cardo C, Zhu J. Identification of microR-106b as a prognostic biomarker of p53like bladder cancers by ActMiR. Oncogene. 2018 Nov;37(44):5858-72.

33-Gowrikumar S, Singh AB, Dhawan P. Role of Claudin Proteins in Regulating Cancer Stem Cells and Chemoresistance-Potential Implication in Disease Prognosis and Therapy. International journal of molecular sciences. 2020 Jan;21(1):53.

34-Nakanishi K, Ogata S, Hiroi S, Tominaga S, Aida S, Kawai T. Expression of occludin and claudins 1, 3, 4, and 7 in urothelial carcinoma of the upper urinary tract. American journal of clinical pathology. 2008 Jul 1;130(1):43-9.

35-Székely E, Törzsök P, Riesz P, Korompay A, Fintha A, Székely T, Lotz G, Nyirády P, Romics I, Tímár J, Schaff Z. Expression of claudins and their prognostic significance in noninvasive urothelial neoplasms of the human urinary bladder. Journal of Histochemistry \& Cytochemistry. 2011 Oct;59(10):932-41.

36-Törzsök P, Riesz P, Kenessey I, Székely E, Somorácz Á, Nyirády P, etal. Claudins and ki- 
Benha medical journal, vol.38, academic issue, 2021

67: potential markers to differentiate low-and high-grade transitional cell carcinomas of the urinary bladder. Journal of Histochemistry \& Cytochemistry. 2011 Nov;59(11):1022-30.

37-Vincent BG, Kim W, Kardos J, Chai S, Parker J, Mose L, etal. The novel claudin-low molecular subtype of high-grade urothelial bladder cancer is highly immunogenic yet immunosuppressed. Cancer Research.2016 Jul;76(14): 51205120.

38-AMR F, EMAN A, EMAN S, YOUSSEF M, SAMIA A. Evaluation of Claudin-1 and Ki-67 in Different Molecular Subtypes of Breast Ductal Carcinoma: Immunohistochemical
Study. The Medical Journal of Cairo University. 2019 Mar 1;87(March):517-26.

39-Dias K, Dvorkin-Gheva A, Hallett RM, Wu Y, Hassell J, Pond GR, Levine M, Whelan T, Bane AL. Claudin-low breast cancer; clinical \& pathological characteristics. PloS one. 2017 Jan 3;12(1):e0168669.

40-Sadalla JC, Lourenço SV, Sotto MN, Baracat EC, Carvalho JP. Claudin and p53 expression in vulvar lichen sclerosus and squamous-cell carcinoma. Journal of clinical pathology. 2011 Oct 1;64(10):853-7.

To cite this article: Eman A. Saad, Kareem A. Ibrahim, Nashwa M. Emaraa, Amr F. ElSebaaie, Eman s. omar, Samia A. Youssef. Role of HER2 and Claudins in Subtypes of Urothelial Carcinoma Identified Bygata3 and Cytokeratin516. Immunohistochemical Study. BMFJ 2021; 38 (academic issue): 128-146. DOI: 10.21608/bmfj.2021.52869.1355 